\title{
Enabling Real-time Information Service on Telehealth System over Cloud-based Big Data Platform
}

\author{
Jihe Wang ${ }^{\mathrm{a}}$, Meikang Qiu ${ }^{\mathrm{b}, *}$, Bing Guo ${ }^{\mathrm{a}}$ \\ ${ }^{a}$ Computer Science College, Sichuan University, Chengdu, SC, China 610064 \\ ${ }^{b}$ Pace University, Seidenberg School of Computer Science and Information Systems, Manhattan, NY, USA 10038
}

\begin{abstract}
A telehealth system covers both clinical and nonclinical uses, which not only provides store-and-forward data services to be offline studied by relevant specialists, but also monitors the real-time physiological data through ubiquitous sensors to support remote telemedicine. However, the current telehealth systems don't consider the velocity and veracity of the big-data system in the medical context. Emergency events generate a large amount of the real-time data, which should be stored in the data center, and forwarded to remote hospitals. Furthermore, patients' information is scattered on the distributed data center, which cannot provide a high-efficient remote real-time service. In this paper, we proposes a probability-based bandwidth model in a telehealth cloud system, which helps cloud broker to provide a high performance allocation of computing nodes and links. This brokering mechanism considers the location protocol of Personal Health Record (PHR) in cloud and schedules the real-time signals with a low information transfer between different hosts. The broker uses several bandwidth evaluating methods to predict the near future usage of bandwidth in a telehealth context. The simulation results show that our model is effective at determining the best performing service, and the inserted service validates the utility of our approach.
\end{abstract}

Keywords: Telehealth, sensor, cloud, bandwidth, real-time.

\section{Introduction}

Global demographic trends to demonstrate a clear rise in the proportion of elderly and chronically ill individuals [1]. At the same time, the strong demand for various medical and public health care services from customer needs the creation of powerful individual-oriented personalized health care service systems [2]. Fortunately, over the recent years, the development of wireless sensor network and cloud computing [3] provides an important solution for delivering patient's Personal Health Record (PHR) and real-time physiological information $[4,5]$. In order to collect the physiological information of patient bodies, ubiquitous Body Sensor Network (BSN) is widely used in many remote health care applications, such as wireless wearable electrocardiography (ECG). Furthermore, based on the scalable computing capability of Data-Center (DC), it is possible to provide the remote diagnosis and medical services to public with high-privacy policy [6, 7]. Current PHR services, such as Microsoft HealthVault and Google Health [8], provide a foundation of adoption decisions and serve as a starting point of requirements analysis for more complicated telehealth systems.

The current telehealth systems have two orthogonal rules to be classified: uses and modes. In order to support the activities of medical and patient, telehealth system should provide both clinical uses and nonclinical uses. For clinical uses system, the teleconference between patient and healthcare provider

\footnotetext{
${ }^{*}$ Corresponding author

Email addresses: wang jihe46@gmail . com (Jihe Wang), mqiu@pace.edu (Meikang Qiu), guobing@scu.edu.cn (Bing Guo)
}

benefits from some cloud services, e.g., transmission of medical images for diagnosis, individuals exchanging health services, and health advice guided in emergent cases (referred to as teletriage). For nonclinical uses system, some distance educating and information managing tasks are performed based on telehealth platforms, e.g., meetings between supervision and presentations, healthcare system integration, and medical/patient education. However, the above uses are the viewpoint of the users (medical and patient), which cannot reveal the key techniques invoked when implementing. Thus, we divide it into store-and-forward mode and real-time mode. For store-andforward mode, digital image, video, audio, observations of daily living and clinical data are captured and stored on cloud or mobile devices; then at a convenient time, they are securely forwarded to a clinic at another location, where they are studied by relevant specialists. For real-time mode, a telecommunications link allows instantaneous interaction. Some typical realtime clinical telehealths include teleaudiology, telecardiology, teledentistry, and telebehavioral health.

Based on the BSN and PHR technologies, DCs and cloud computing are supporting the information storage and delivering services for medicals and patients. For example, the highlevel medical services, such as disease early warming and preliminary decision making, could be implemented on cloud end or hospital end. In addition, the QoS and data security can be both guaranteed with cloud, where, privacy data are redundantly stored with encryption algorithms [7]. All of the these applications constitute a big-data system to serve the remote individuals. The efficiency of big-data within neonatal inten- 
sive care units is reported to have a great potential to support new wave of clinical discovery, leading to earlier detection and prevention of wide range of deadly medical condition [9].

In a distributed data coherence system, when source node is executing a service, it might continually fetch the latest PHR information from target nodes. As a distributed system, telehealth services meet two kernel problems for data coherence between remote nodes. Firstly, the computing source nodes may not be the home node of the PHR, which means locating data is a slow procedure by inquiring to the home node. Secondly, the coherence protocol fails to guarantee the data synchronization of multiple copies of one PHR in the distributed system, which cannot help its cache system to increase the performance and hit-rate. The traditional memory solution may lead to significant increasing of the queue delay time of the waiting jobs with normal memory requirement, which slows down the execution of each job and decreases the system throughput $[10,11]$. In this work, we adopt the store-and-forward and real-time modes as the basic division to the telehealth services. The former includes all medical applications that have to refer the patient's personal information and disease history. Indeed, the PHR information could reside in any location of the cloud. The later [12] always collect the patient's current physiological signal, such as ECG, from local BSN, and transfer the information to cloud. Hence, one copy of the real-time signal is given to the remote medical for monitoring through external network, and another copy, as the historical record, should be saved in the host DC of the patient.

In a distributed computing and storing system, cloud broker takes charge of both the service request from remote BSN and the resources allocation on cloud. As one of the most important components, broker introduces the incoming cloud consumers to health service providers so that the resource could be utilized with a higher efficiency and balance. Unfortunately, the most of distributed brokers only monitor the resource information, such as utilization of CPU, memory, bandwidth, and disk space in each node. The location information of the multiple PHR copies is transparent to brokers, which blocks brokers to make a wise scheduling to allocate computing task near the available PHRs. This invisible of PHR information results in that broker cannot choose the high-performance node and link for the PHR data transferring between the home and target node. Traditional data coherence protocol on application layer, such as MESI protocol [13], also ignores the real location of specific PHR data, which also failed to suggest broker which node should be chosen.

The work in this paper aims to adding a content-aware service to cloud broker so that the location of PHR copies can help broker to allocate proper bandwidth to DCs with high predictability and networking efficiency. Our main contributions are:

1 We design a data coherence protocol for the distributed system, where the PHRs can be stored and accessed by distributed data centers with a high performance.

2 Based on the protocol, a flow estimating algorithm is proposed to evaluate the bandwidth consumption on each data center by the real-time signal from the remote BSNs.

3 We design several predicting methods for the future bandwidth consumption, based on which, bandwidth allocating algorithms can be applied to various applications with different features in the bandwidth leasing system.

The rest of the paper is organized as follow. The Section 2 includes some related work. An overview of the architecture of our cloud-based telehealth platform is proposed in Section 3. Section 4 explains the details of our traffic load model for remote data accessing. Section 5 presents our model about calculating the traffic load of real-time signal based on the data coherence protocol. Some traffic prediction models are illustrated in Section 6. The implementation and experimental results of the platform are reported in Section 7 and 8. Finally, Section 9 concludes the paper.

\section{Related Work}

To provide cloud-based health care system has triggered considerable researches both in industry and academia in these years. In October 2011, Microsoft released a new version Webbased platform, HealthVault, to help users to gather, store, use, and share health information for them and their family [8]. In $[14,15]$, the authors described the prototype for remote health care monitoring, and considered usability, power supplies, and expensive issues in the context of localized multi-sensory wearable networks and presented a method to generate low-power sampling schedules which were resilient to sensor faults while achieving high diagnostic fidelity. Paper [1] provided an approach involving telehealth applications, many of which were based on sensor technologies for unobtrusive monitoring. The importance of these applications was considerable in light of the global demographic trends and the resultant rose in the occurrence of injurious falls and the decrease of physical activity.

In [2], the authors presented PHISP (a Public-oriented Health care Information Service Platform) which supported numerous health care tasks, provided individuals with many intelligent and personalized services, and supported basic remote health care and guardianship. Paper [3] proposed on-line dynamic resource allocation algorithms for the IaaS cloud system with preemptable tasks, which adjusted the resource allocation dynamically based on the updated information of the actual task executions. To provide a security representation of PHR, XML schema is adopted in work [16]. Also, the PHR representation supports some data mining techniques for further clinical studies [17]. The previous solution cannot strain there performance with the BSN workload because they invoked negative response at the serving end.

Despite sensor network in the area of telehealth service, much efforts have specifically focused on the traffic load balance for power, storage space and energy saving which introduces some control structures in the process models for cloud. In the work of [18], each virtual node itself decided whether to replicate, migrate or suicide by weighing up the pros and cons, which was based on the evaluation of traffic load of all nodes, 
and selected among physical nodes with the most traffic to replicate or migrate on. After that, it takes into account blocking probability to achieve quicker response and better load balance performance. In [19] and [20], the authors presented the realtime virtualised cloud infrastructure that was developed in the context of the IRMOS European Project. The work in [21] proposed an interface design of a low-power programmable system on chip for intelligent wireless sensor nodes to reduce the overall power consumption of the heart disease monitoring system. Those work didn't provide service with the requirement quantities, and no model was applied within them to guide a proper performance configuration.

\section{System Architecture}

As a cloud-based telehealth system, the real-time signal should be collected by distributed context-aware BSNs and transmitted to the DCs which stores and forwards the signal for immediate and future usage. In addition to providing continuous monitoring and analysis of physiological parameters, BSN incorporates context aware sensing for increased sensitivity and specificity. The typical devices include ECG, blood pressure monitor, glucose meter, pulse oximeter and so on [22, 23]. By adopting the IEEE 802.15.4 standards [24], sufficient bandwidth, low-power, and low-cost personal area network is available for demanding continuous physiological and context sensing.

To implement a BSN, a number of wireless biosensors including 3-lead ECG, 2-lead ECG strip, and oxygen saturation (SpO2) sensors connect together with a hub Personal Digital Assistant (PDA). The hub PDA can also act as the router between the BSN nodes and the central server, where all sensor data collected will be transmitted through a WiFi/GRPS network for long-term storage and trend analysis [25]. For example, an ECG sensor with signal conditioning and processing circuits is capable of providing useful vital signal on heart functioning. The signals coming from electrodes must be amplified and filtered in order to generate an appropriate low-noise signal that fits within the input range of the Analog-Digital Converter (ADC) and data rate of the transceiver [26]. To capture clinically relevant episodes, a group of context aware sensors are incorporated into the BSN, and the hub PDA is used for multisensor data fusion before transmitting the data to the cloud DC with minimal bandwidth.

The real-time data produced by those sensors is a significant workload for each node in DCs. Firstly, medical applications on cloud monitor individuals' physiological signals and analyze their characters to determine the healthy status. If an individual is found to be in emergency, alarm message is sent to relevant medical entities. Secondly, individual's physiological signals need to be stored and copies in the storage system on cloud, which aggravates the performance of disk-based storing. Actually, the physiological signal in telehealth system has no difference from other types of real-time signal in nature. However, we should limit its impact on the performance of DCs.

Figure 1 illustrates the overview of our cloud platform architecture. The infrastructure of our health care cloud platform is mainly composed with BSNs, brokers and some distributed

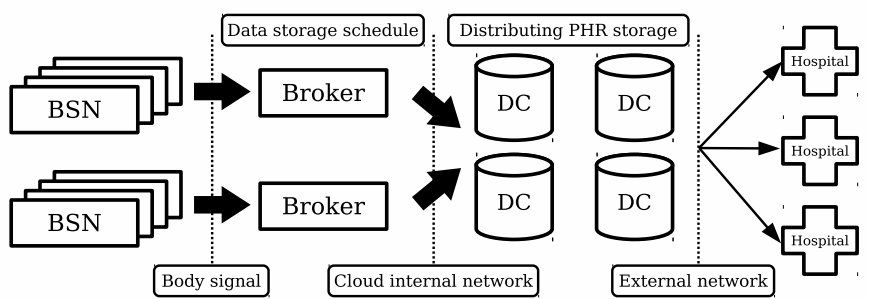

Figure 1: The architecture of the cloud platform.

DCs. It's difficult to evaluate the performance of cloud policies, application workload models and resources performance models in a repeatable manner under varying system, user configuration, and requirements [27, 28].

1 The front end BSN centers, such as DSP and patient's computer, are the clients of the cloud computing tasks. The BSNs generate the task request to broker through the Internet and obtain the authentication from cloud to utilize the service. And each BSN can be treated as a data source in our model.

2 The brokers are responsible to reserve an appropriate DC for the service and deliver the request to the DC. As the cloud manager, brokers received the BSN request from remote and allocate the bandwidth resource to the BSN data stream.

3 In the distributed DCs, some health care services are set up, such as PHR repository and scientific knowledge base of health care, and personal health applications acquired by BSN. These jobs always involve a large amount of parallel task, which can be classified by the terms like high-throughput computing (HTC) or many-task computing $[29,30]$. At the same time, there are several copies of PHR, in the distributed DCs, which need to be synchronized with data coherence protocol.

There are three types of node in the system to support a 3-way data transmitting: request node, home node, and target node. We list their definitions in follow.

Request node. A request node in a DC is a broker which plays two roles, i.e., 1) a broker receives the data transmitting requests from BSNs, and 2) a broker finds the appropriate nodes to process and store the BSN data stream.

Home node. A home node for a PHR is the node which stores the current status of the PHR data, such as the PHR's locating information, accessing permission, and the synchronizing information. With the home node, querier is able to determine how to find the PHR data on a distributed system. Notice that one piece of PHR is mapped to only one home node. We will give a mapping model in the next section.

Target node. A target node for a PHR is the node which have a copy of the PHR, no matter the PHR is the latest version. In big-data, each PHR should have at least the same copies at three different target nodes. 


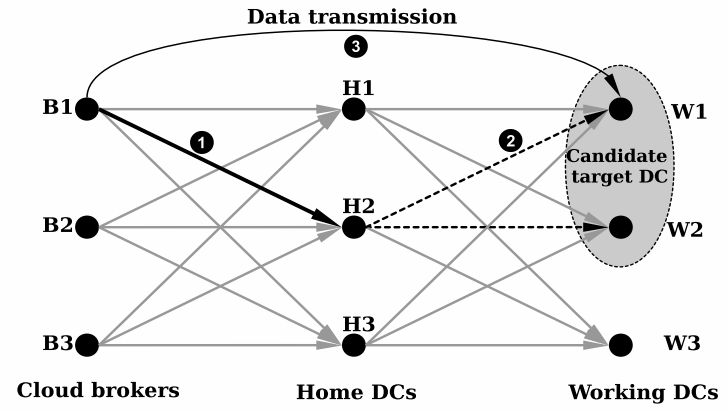

Figure 2: The procedure of setting up a real-time connection. The 3-way data connection includes: 10 a random broker, says B1, accepts the data transmitting request from remote BSN and the patient's PHR points to one of the Home DCs, says H2, 2 the Home DC looks up its local table and a set of candidate target DCs are filtered out, says W1 and W2, with specific algorithm in the home node, and $\mathbf{3}$ one working DCs in the candidate set is chosen as the server for the request and the real-time data transmission and processing jobs is assigned to the server, says W1.

\section{Data Coherence Protocol in Cloud}

The current management method on cloud features a single centralized node that is dedicated to respond all the data request from brokers, referred as name-node [31]. This kind of data management has two drawbacks that 1) the name-node has to trace the changes and movements of all PHR data with high CPU and networking utilities, and 2) inquiring data location in a single big mapping table is a time-consuming task. In this section, we describe the detail of the data coherence protocol that supports the remote data accessing in the distributed data centers, which is able to overcome those drawbacks with centralized design.

\subsection{The definitions of the Data Coherence Protocol}

In a distributed system, each memory module is associated with a group of DCs, and each DC can directly access its own memory. Thus, a message passing mechanism is used to access remote memory modules in the cloud system. A directed graph $\operatorname{Cloud}\left(S_{N}, L\right)$ is used to model a cloud system with $N$ DCs, where $s_{i} \in S_{N}$ is the $i$ th DC in the graph, $i$ represents the location of node $s_{i}, 0 \leq i \leq N-1 . l_{i, j} \in L$ is a directed edge between $s_{i}$ and $s_{j}$. A one-dimensional flat PHR can be expressed as a two-tuple $P H R\left(v, d c_{-} v\right)$, where $v$ is the PHR volume of the cloud memory space and $d c_{-} v$ is the PHR volume of single DC, in which, a distributed directory record table maps all PHR to the local memory and stores their current states. We design a mapping function from a PHR ID phr_id in memory to its home node's location $i$ in Cloud as follow.

$$
i=\left\lfloor\frac{p h r_{-} i d}{d c_{-} v}\right\rfloor
$$

We describe the procedure of bandwidth allocation as shown in Figure 2:

Request step. A request node is a broker node which utilizes the locating function to determine the home node of the patient's PHR. The request node sends a request message with patients' ID information to the home node.

Response step. After the home node receives a request message, it hashes the patient's ID to a memory address from which the owner list of the patient's PHR can be obtained. The home node produces a probability matrix about task distribution in the cloud, and sends the matrix back to the request node. This matrix is introduced in Section 5.

Transmitting step. After the home node receives the probability matrix, it determines the most appropriate DC as the signal's target node. Therefore, a real-time physiological signal from BSN can be transmitted through the request broker node $\rightarrow$ target node $\rightarrow$ hospital server path.

\subsection{The PHR Delivery among Data Centers}

The Figures 3 (a) and (b) illustrate the procedures of the reading and writing on remote target node, respectively. The principle of the design is that the home and target node should provide a transparent service for the request node. In Figure 3 (a), at the beginning, the request node sends a request message to the home node with the function of Equation 1. When the home node finds that there is a available data (PHR) copy in a target node, it multicasts two messages to the request and target nodes. The function of the multicasting is to transfer the current communicating pair to the request and target nodes. After that, the target node gives a response message to both of the request and home node, so that the remote data accessing can start. Finally, the request node notices the home node that it has received a new copy of the data, and the home node changes the status of the data in its local record table.

In the implementation, we use state machine to control the states of each node, as shown in Figure 4. The detail description of these states are listed as following.

$r \_R E A D \_W A I T$ : The request node has sent a REQ_S YN message to home node, and is waiting for the response.

$r_{-} T A R_{-} W A I T$ : The request node is waiting for the response message from the target node for starting a data transmission.

$r \_$RECEIVING: The data transmission is set up and the data is copied from the remote space to a local memory.

r_READ_CLOSE: The data transmission has finished.

h_READ_WAIT: After home node sent one $R E Q$ : $A C K$ message to the request node, and another REP : $S Y N$ message to the target node, the home node is waiting for the response of target node.

$h \_$RECEIVING: The home node is waiting for finishing the data transmission.

$t \_R E A D_{-} W A I T$ : The target node has received a request message from the home, while it is waiting for the start-up of the data transmission from the request node.

$t \leq S E N D I N G$ : The data is copied from the local space to the remote request node.

FAIL_REQ: The home node cannot find any record of available data copies in the distributed storage system.

When the three nodes complete one round of the data transmission, all of them will return to their start nodes, and waiting for new coming data transferring demand. 


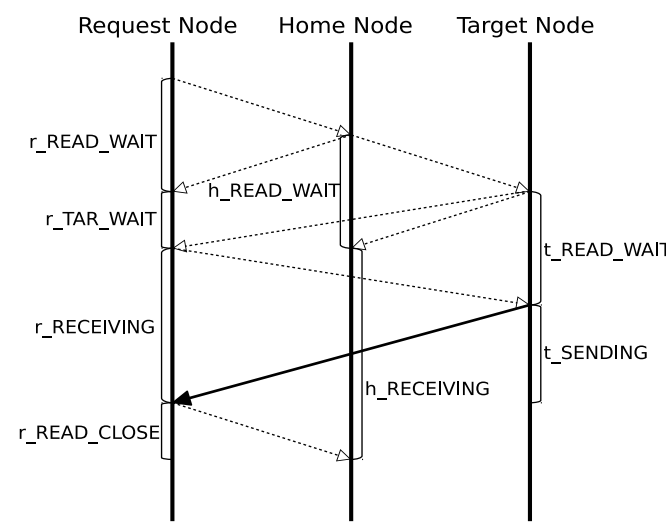

(a) The connections of reading data from remote node.

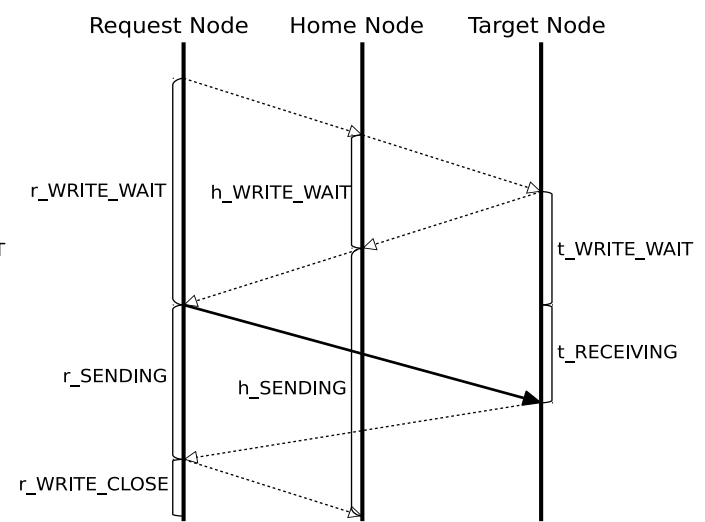

(b) The connections of writing data to remote node.

Figure 3: The diagram of reading and writing connections among nodes.

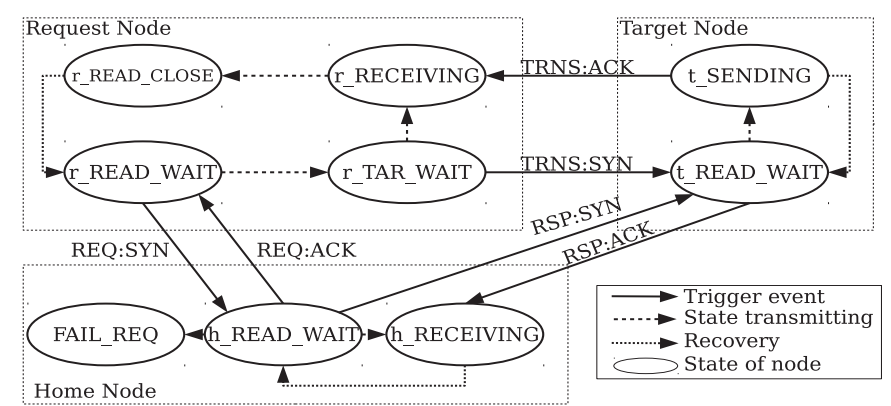

Figure 4: The state machines of each node during a remote accessing procedure. The three sub-state-machines cooperate to implement the transmitting protocol. For example, the Request node needs to trace the current location of a task at Home node or Target node. When the task is searching its candidate nodes in the Home node, the Request node has to stay in "r_READ_WAIT" state. When the task is in Target node, the Request could go to the next state, like "r_TAR_WAIT", for further data transmission.

After a request node got a new version of the data, the data should be sent to the target node so that the newest data can be used in future access on cloud. Figure 3 (b) shows the detail of this procedure. When the request node sends a request message to the home node, the home node should notice the target node that there will be a new copy of the data from remote node. Then, the target node responds the home node with a respond message, which is further forwarded to the request node to set up a communication between the request and target nodes. After the new version of data is transmitted to the target node, a "CLOSE" message is passed to the home node that would like to change the status of the data in its local record table. Generally, the updating speed of the record table could be the majority bottleneck of the system. Our test shows that updating workload is positive linear with the BSN workload. The phenomenon further confirm that the sample-based design can really reflect the system workload.

The protocol defined in this section doesn't conflict with some status-based data coherence protocols, like MESI. Our protocol gives the data transmitting protocol between different nodes, and the MESI only shows the accessibility of PHR data pieces. In the context of telehealth, the real-time data has an important feature that the signal data is always single-written and multi-read by users. Therefore, the accessibility is not in the primary issues since there is not writing-conflict, provided that the user gets operating permission.

\section{Traffic Load Model of Data Coherence}

In this section, we illustrate the details of our traffic load model. In previous section, we divide the a typical real-time stream service into two parts. One is the PHR related service which provides the information coherence service and guides the real-time signal to be routed to proper nodes; another is the real-time related service which completes the signal transmitting to the remote medicals. In this work, a probability-based traffic load model is designed and inserted into the cloud broker. This model accepts the feedback information from each data center and makes the decision of resource schedule.

\subsection{Distribution of Request and Response}

In this cloud system, all request messages requiring patient's PHR information can be seen as random events from BSNs. Thus, we use $q_{i, j}$ to represent the probability of broker $_{i}$ 's PHR asking for a piece of PHR item whose home node is node $j$. Then we utilize a matrix $\mathbf{Q}$ to describe the request sending probability distribution of the whole cloud system. Specifically, 1) If the diagonal elements $q_{i, i}>0$, one portion of requests from cloud node $i$ hit the broker's local DC node, and node $i$ does not need to look up the directory item in remote nodes. If $q_{i, i}=0$, the required PHR data is always found on the remote DC. 2) Since $\mathbf{Q}$ is a probability matrix, it is natural that the sum of a row in $\mathbf{Q}$ must equal 1. 3) $q_{i, j}(i \neq j)$ could be any value between 0 and 1. Matrix $\mathbf{Q}$ is a probability matrix that contains more hints about the data transmission. If requests from the broker node $i$ are mainly requests to nearby nodes rather than to distant ones, the bandwidth consumption of the whole cloud system could be lower. For example, in a $2 \times 2$ cloud system, consider a probability vector $\mathbf{q}=\left(q_{1,0}, q_{1,1}, q_{1,2}, q_{1,3}\right)$ which is one row of the $4 \times 4$ matrix $\mathbf{Q}$. If the probability of this row 
is mainly contributed by $q_{1,0}, q_{1,1}$ and $q_{1,2}$, then the traffic load from the requests broker to distant DC nodes, $q_{1,3}$, cannot affect the available bandwidth of other link significantly. However, if the value of $q_{1,3}$ is dominant, the requests from node 1 to node 3 may seriously impact overall bandwidth of the cloud system.

We adopt a similar approach to describe the response probability distribution. A matrix $\mathbf{P}$ can be used to express the relationship between home and target DC nodes. Its element $p_{j, k}$ is the probability with which the home node $j$ sends a new copy of the PHR to the target node $k$, no matter whether the data's copy has been in the target node. In some specific implementations of the coherence protocol, designers employ different swap-out methods to delete the trashy image or spread a PHR's image in a limited range, where some data can never appear in a node or will always be accessed by a node. These optimal strategies are viewed as special probability distributions in our matrix space, which may lead to better performances, such as fewer average transmission hops and lower bandwidth impact. We do not intend to restrict this model in a specific implementation, so it is reasonable to assume that a copy of PHR can resident in any DC node, and a specific strategy always has corresponding probability distribution matrix $\mathbf{P}$.

\subsection{Algorithm of Traffic Load Distribution}

For data coherence protocol based memory systems, synchronization is always a costly operation. A request broker node has to look up the directory items stored in the home DC node before a data transmission action starts. That means, on the one hand, the requester doesn't know which DC node is available for a copy of the PHR data in cloud until the search finishes in the home node. On the other hand, when a DC node is searching in its directory table, it doesn't need to refer the location of the request broker node on the network. If we use $R E Q_{i, j}$ to represent the random event that manager node $i$ sends a request to DC node $j$, and $S E A_{j, k}$ to represent the random event that node $j$ finds the needed data to store in node $k$, then the events $R E Q_{i, j}$ and $S E A_{j, k}$ are statistically independent from each other:

$$
\operatorname{Pr}\left(R E Q_{i, j} \cap S E A_{j, k}\right)=\operatorname{Pr}\left(R E Q_{i, j}\right) \operatorname{Pr}\left(S E A_{j, k}\right)
$$

In some data coherence protocol implementations, several copies of one cache block may be distributed in different DC nodes, as shown in Figure 2. These nodes constitute a candidate set. Any node in this set could be a potential target node in the transmitting step. We only select one node located closest to the requester in order to shorten the transmission distance, which could leads to lower bandwidth consumption. Since the candidate set is independent from the location of the requester node, the selected target node's position is also independent from the requester, no matter how close the target node will be from the request node.

The final goal of the design is to send the real-time physiological signal from the cloud manager node to the request DC node. Considering that if a real-time signal is transferring from broker node $i$ to DC node $k$, two independent events, $R E Q_{i, j}$ and $S E A_{j, k}$, have happened before the data transfer. The probability of a signal transmitting from node $i$ to node $k$ can be formulated as:

$$
\operatorname{Pr}\left(S I G_{i, k}\right)=\sum_{j=0}^{N-1} \operatorname{Pr}\left(R E Q_{i, j}\right) \operatorname{Pr}\left(S E A_{j, k}\right)
$$

where $S I G_{i, k}$ is the event that a signal transfers from manager node $i$ to DC node $k$. We use an $N \times N$ matrix $\mathbf{T}$ to represent the probability distribution of signal transferring, then $\mathbf{T}$ can be deduced from Equation (3) as:

$$
\mathbf{T}=(\mathbf{Q P})^{T}
$$

The elements of matrix $\mathbf{T}$ are similar to that of $\mathbf{Q}$ and $\mathbf{P}$. Element $t_{i, k}$ is the probability of sending a packet from broker node $i$ to DC node $k$. Algorithm 1 shows the distributed procedure forming the signal transmitting matrix $\mathbf{T}$.

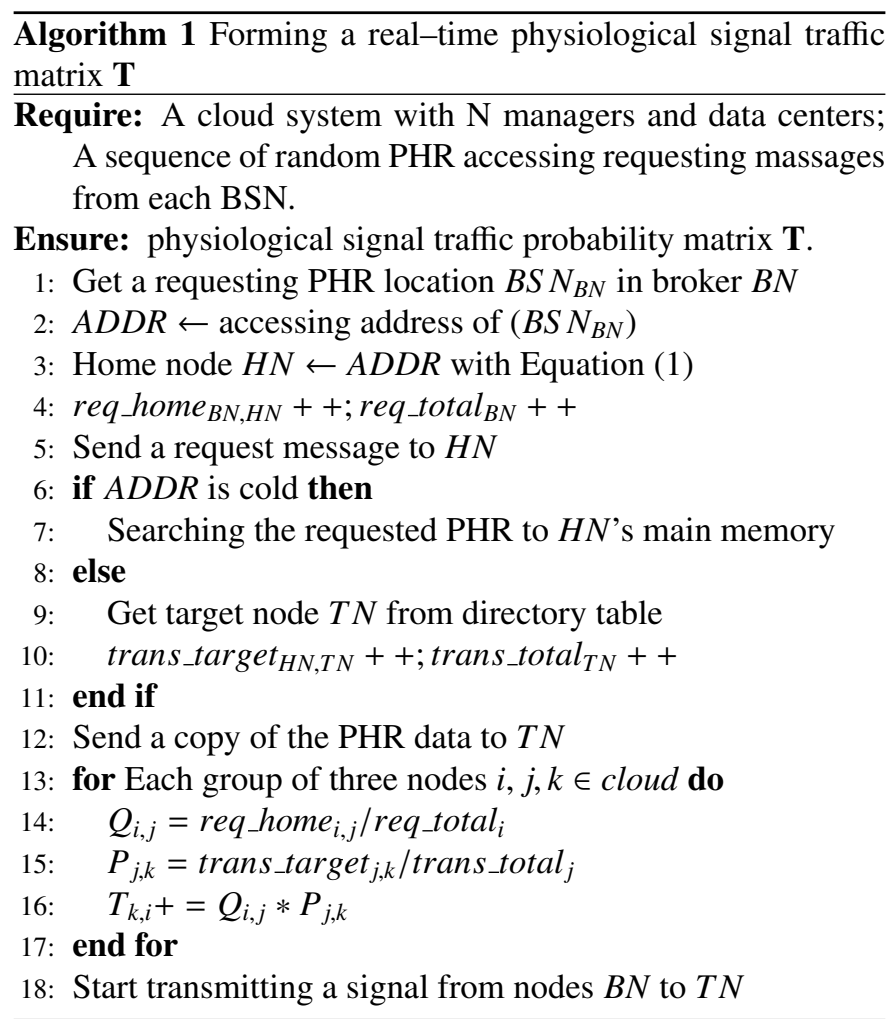

In the Algorithm 1, the lines 1-4 show the procedure of calculating the address of home node in cloud. A broker receives a request from a BSN and extract the PHR ID from the request (lines $1-2$ ). Then a locating function is invoked to produce the address of the PHR (line 3). Line 4 updates the request numbers of each DC node and total cloud. After the above procedure, a request massage is sent to the Home Node $(H M)$. Lines $5-12$ describe the PHR fetching procedure in home node. If the PHR is cold, which means this PHR directory is not cached the home host, the host should schedule a virtual machine to search the PHR information in the main memory. Otherwise, the target node can be extracted from the cached directory table quickly, and some updating work is applied in line 10. In line 12, a copy of the PHR data is sent to the target node. Lines $13-17$ are the iteration to update the elements of the matrix T. And this 
algorithm only takes the computing complexity of $O(\log (N))$, where $N$ is the quantity of active users.

We define $b_{i, j}$ as the bandwidth consumption by a real-time signal transmission from node $i$ to node $j$. Thus, $b_{i, j}$ could be a fixed parameter of the shortest path between a pair of nodes in the cloud. We multiply $b_{i, j}$ to each element of the PHR transferring probability matrix $\mathbf{T}$ shown in Equation (5), and get the bandwidth consumption distribution by the real-time signal service.

$$
B=\left(b_{i, j} t_{i, j}\right)_{N \times N}
$$

The matrix $\mathbf{B}$ can be used to display the bandwidth consumption distribution of data transferring in the cloud system. In particular, 1) The sum of a row $\sum_{i=0}^{N-1} b_{k, i} t_{k, i}$ is the expected bandwidth value by sending a physiological signal from manager node $i$ to data center node $k$. 2) The diagonal element $b_{k, k} t_{k, k}$ equals 0 , which means $b_{k, k}=0$, because a DC won't consume network bandwidth when it sees itself as both home and target node at the same time.

\subsection{Bandwidth Optimizing Scalar}

To use our traffic model to evaluate different PHR organizing and task delivering algorithms, we present a random signal solution that does not include any optimizing information. By comparing with the random sending solution, we can determine the optimization gain of a given solution. Using the expected bandwidth of a single signal between a broker node and DC node, we define a random sending solution which is a complete, fair packet sending instance that satisfies $q_{i, j}=q_{i, k}$ and $p_{i, j}=p_{i, k}$ for any node identifier $i, j$, and $k$. In a random sending solution, all elements in matrix $\mathbf{P}, \mathbf{Q}$, and $\mathbf{T}$ equal $1 / N$, where $N$ is the node quantity in the cloud, which comes from $q_{i, j}=p_{j, k}=1 / N$ for any node $i, j$, and $k$. Then $t_{i, k}=q_{i} p_{k}=$ $N \times(1 / N)^{2}=1 / N$, where $q_{i}$ and $p_{k}$ are the $i$ th row vector of matrix $\mathbf{Q}$ and $k$ th column vector of matrix $\mathbf{P}$, respectively.

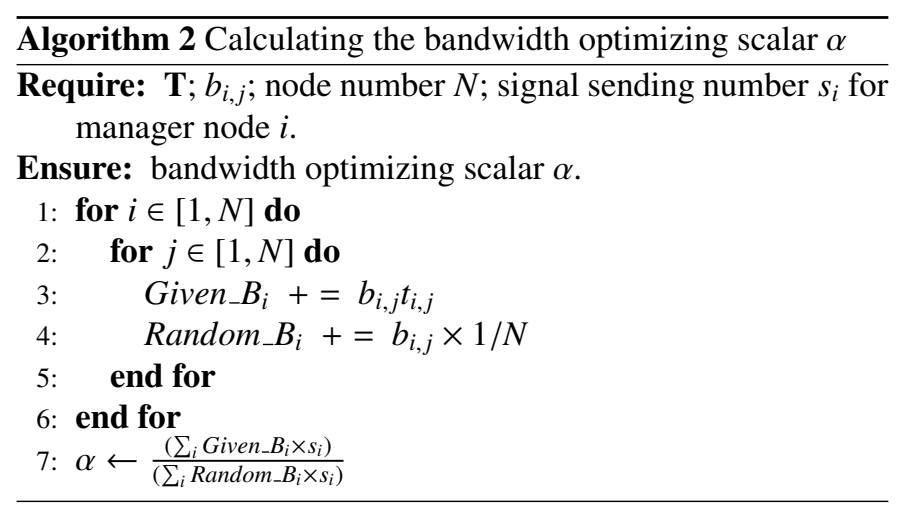

Algorithm 2 gives the function of getting the bandwidth optimizing scalar $\alpha$. For each pair of nodes $(i, j)$, the algorithm calculates a new available bandwidth of both given and random sending solutions in lines $3-4$. Finally, the optimizing scalar $\alpha$ is produced in line 7 . In the given sending solution, matrix $\mathbf{T}$ is used to define the signal sending probability distribution for each broker node. In the random sending solution, the sending probability between any pair of manager and data center nodes
Table 1: Various bandwidth predicting methods.

\begin{tabular}{|c|c|}
\hline Name & Function \\
\hline AP & $t_{i}^{1}=\frac{1}{m} \sum_{s=1}^{m} t_{i}^{1-s}$ \\
\hline $\mathrm{WP}$ & $t_{i}^{1}=\sum_{s=1}^{m-2}\left(2^{-s} t_{i}^{1-s}\right)+2^{1-m}\left(t_{i}^{1-m}+t_{i}^{-m}\right)$ \\
\hline $\mathrm{LSP}$ & $t_{i}^{1}=\beta_{0}+\beta_{1} x_{i}$ \\
\hline $\mathrm{RP}$ & $t_{i}^{1}=\frac{1}{m} \sum_{s=1}^{m} t_{i}^{1-s}+\xi(\hat{\mu}, \hat{\sigma})$ \\
\hline
\end{tabular}

is uniform, so there is no information about data aggregation and address optimization on this solution. It's unnecessary to adopt a solution whose bandwidth consumption is worse than the random solution. A lower $\alpha$ indicates that the given solution can save more bandwidth during the transmission of a signal from the manager node to the target data center. As a feedback parameter, the bandwidth optimizing scalar $\alpha$ can be used to choose the best target DC, which is able to make minimal bandwidth impact on the traffic efficiency of the whole cloud system.

\section{Traffic Load Estimation Algorithms}

With the traffic load model in Section 5, cloud managers are able to quickly determine the best target data center as the target node in the cloud system. In this section, we use the traffic load matrix to make prediction which guides cloud managers to find an available data center for signal storage and forwarding, and an integral work flow of choosing procedure is also presented. As mentioned in Section 5, the matrix $\mathbf{T}$ conveys the real-time signal distribution on the multi-cloud system. The $i$ th row of T's elements, vector $t_{i}=\left[t_{i, 0}, t_{i, 1}, \ldots t_{i, N-1}\right]$, is the probability distribution of the event that cloud manager $i$ sends real-time signals to each data center node. However, as a statistical variables, the vector only displays the distribution in a period of time. That means, we can consecutively record the vector to make the bandwidth prediction in the future. For a cloud manager $C M_{i}$, we record the probability vectors in last $m$ intervals: $t_{i}^{-m+1}, t_{i}^{-m+2}, \ldots, t_{i}^{0}$. Note that a scalar sequence $\left(t_{i}^{-m+1}\right)_{k},\left(t_{i}^{-m+2}\right)_{k}, \ldots,\left(t_{i}^{0}\right)_{k}$ is the historical records of signal sending probabilities from $C M_{i}$ to $k$ th data center $\left(D C_{k}\right)$. There are several simple predicting functions to guess the future probability values which should be normalized so that the result vector is also a probability vector: Average Prediction $(A P)$, Weighted Prediction (WP), Least Squares Prediction (LSP), and Random Prediction (RP). We list their functions in Table 1.

\subsection{Fundamental Bandwidth Predicting Methods}

Average Prediction (AP). The simplest prediction method is to produce an average values of previous $m$ intervals. The basic assumption behind the averaging prediction is that the bandwidth by real-time signal is "locally stationary" with a slowly varying mean. A small value of $m$ will have less of a smoothing effect and be more responsive to recent changes, while a larger $m$ will have a greater smoothing effect, and produce a more pronounced lag in the smoothed sequence. The results of 
the average function need not to be normalized because an average function keeps the "sum-in-one" feature of a probability vector.

Weighted Prediction (WP). A weighted predicting method gives a more flexible way to control the importance of previous bandwidth. Its idea comes from the common phenomenon that a recent bandwidth values could be more important than old values in the prediction. So in practice the weighting factors are often chosen to give more weight to the most recent values and less weight to older values. At the same time, the weight vector $\left\{w_{1}, w_{2}, \ldots w_{n}\right\}$ must satisfy $\sum_{s=1}^{n} w_{s}=1$. We choose $w_{s}=2^{-s}$ when they are not the most distant two terms, and $w_{s}=2^{1-m}$ when they are the rest two terms. For example, if the cloud manager keep 4 intervals bandwidth value from the traffic matrix in Section 5.2, the weight vector could be $\left[\frac{1}{2}, \frac{1}{4}, \frac{1}{8}, \frac{1}{8}\right]$. The weighted predicting function does not keep the normalization feature, so the results should be stretched for a "sum-in-one" vector.

Least Squares Prediction (LSP). The method of least squares is a standard approach to describe the trend of a value sequence. The main idea is to minimize the sum of the squares of the errors made in the results of every single equation. The minimum is determined by calculating the partial derivatives of a sum of squares of "errors" functions. In the expression of Table 1 , the parameters $\beta_{0}, \beta_{1}$ comes from the previous $m$ stages vectors. The least squares method cannot give a normalized result because the predicting values lost their dependence in a same vector.

Random Prediction (RP). Our random predicting model comes from an evolution of the average prediction. It adds a Gauss random error onto the average predicting results, which make the model have a chance to guess the bandwidth in future. The error has the average of $\mu$ which is the average of previous $m$ vectors, and variance of $\sigma^{2}$ is the statistical variance of previous $m$ vectors. In practice, the introduced error could lead to that a probability vector includes some negative elements which is unacceptable as an estimator. To solve the problem, we limit the range of error to $\pm 50 \%$ of the average value by replacing the out-of-range value with a uniformly distributed various within the range. The random predicting method does not guarantee the normalization feature of the result vector, yet.

After gaining the predicted probability distribution vector, it's convenient for the cloud manager to select the data center with lowest bandwidth excepted, which provides a greedy method to balance the real-time signals in the cloud system. The greedy method records the bandwidth usage history between each pair of node and uses the predicting method in Table 1 to calculate the bandwidth allocation in future. The more details about the estimation has been in our previous work [32].

\section{Implementation}

We discuss the performance of our algorithm in the context of a multiple cloud system which contains 16 distributed DCs. In the $4 \times 4$ interval mesh network, each pair of neighbor nodes are connected with a link which has $100 \mathrm{Mbps}$ bandwidth which is not enough when most of remote BSNs request a realtime transmission simultaneously. Each DC is connected with a cloud broker which receives the real-time signal and allocates bandwidth for incoming requesting for patient. We use $256 \mathrm{Vir}$ tual Machines (VMs) to mimic BSNs' action. They produce urgent conditions randomly and send service request and realtime signal to cloud brokers. Each of the VM is controlled to send the request with an increasing probability in an interval. For example, when the interval is set to 1 second, the expectations of bursting probability $P(B P)$ and idling probability $P(I P)$ of a BSN node satisfy $P(B P)+P(I P)=1$. Each real-time signal is set to consume fixed bandwidth of $2 \mathrm{Mbps}$. The brokers response the request by the work flow in Figure 5. The scenarios illustrate the potential use of the prototype we introduced at Section 3. We design three different PHR data coherence protocols to evaluate different task mapping strategies of the data synchronizing procedure: small, middle, and big coherence protocols. For the small data coherence protocol, a small area around the home data center node is priority to be synchronized with the newest PHR data, such as the nodes in a $1 \times 2$ rectangle area. For the middle and big coherence protocol, the area are set to $2 \times 2$ and $4 \times 4$ square around home node, respectively. The basic idea of this design is that, if a PHR is mapped to several neighbor nodes, the communication between the neighbors will happen with much higher sending probabilities than the remote node pair.

Basically, a typical PHR should contain the important information and historical records, such as emergency contacts, health insurance information, current medications and dosages, important hereditary conditions in family history, or even any information the patient wants to include about his/her health. In this implementation, we use extensible markup language (XML) to store and transmit patient's PHR information. As a data coherence design, the simple MSI protocol can guarantee the coherent data accessing in the distributed data center system which always stores multiple copies of a PHR file.

\section{Experimental Results}

The services of traffic load description and estimation method in Section 5 and Section 6 display the work flow of the typical telehealth application. For our purpose, we create and test a new cloud broker following the presented approaches.

\subsection{The Accuracy and Stability of Model}

One of the factors affecting the performance of the model is the frequency of the request from the BSNs. In this section, we also use the $256 \mathrm{VMs}$ to simulate the requests from BSNs. Each of the VM sends the request to the cloud manager with a specific rate.

The error of a prediction model can be defined as:

$$
\text { error }=\frac{\mid \text { predict }- \text { measure } \mid}{\text { measure }}
$$

We explore the sending rate from 0.1 to 0.9 . Figure 6 shows the relative model errors for the three protocols. For the big protocol, the maximal model error, $9.3 \%$, comes from the scenario 


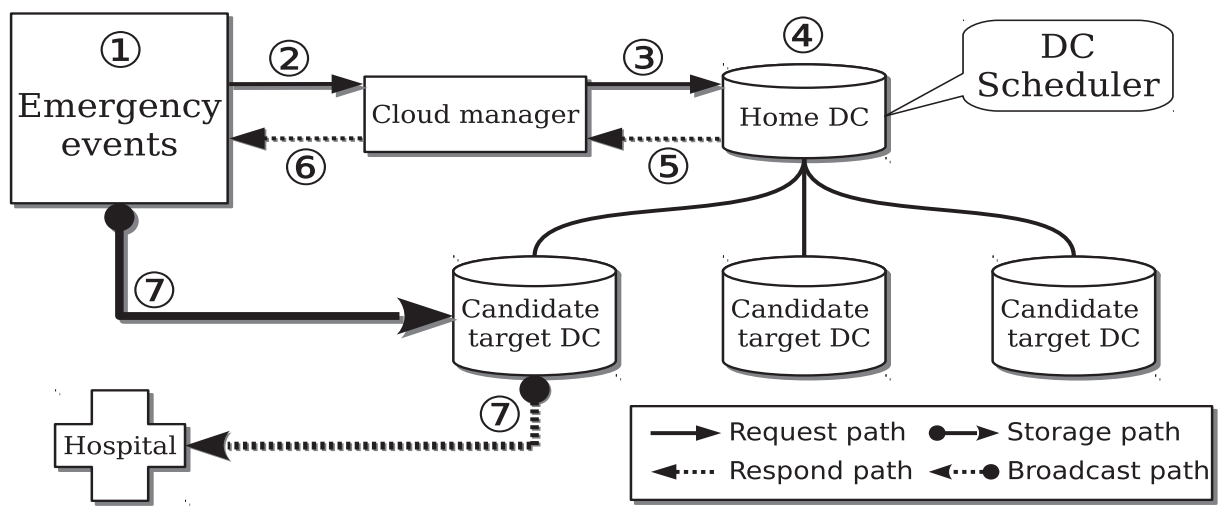

Figure 5: The work flow of the cloud system for telehealth: (1) An emergency event occurs in BSN; (2) the BSN sends request to a cloud manager; (3) the manager queries home node current status of PHR; (4) the home node searches in local storage; (5) the home node returns the PHR's locations; (6) the manger asks for a real-time signal; (7) the real-time signal is stored in one of DCs and transmitted to hospital servers.

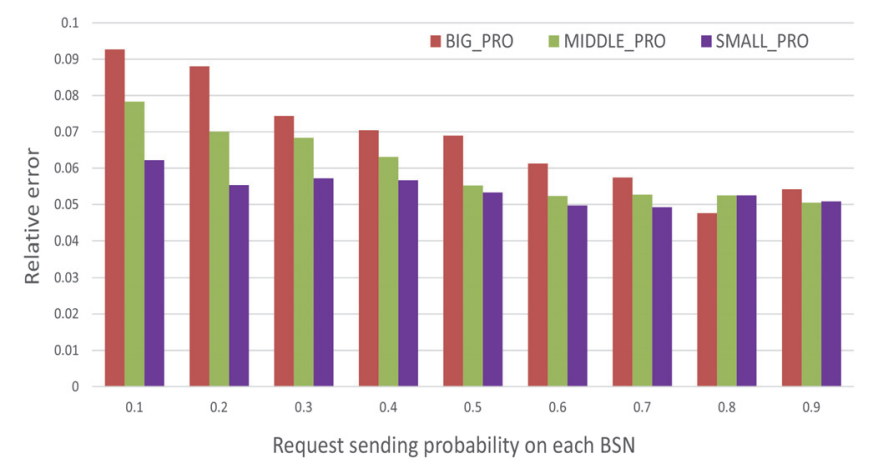

Figure 6: The request sending probabilities and model errors for each protocol.

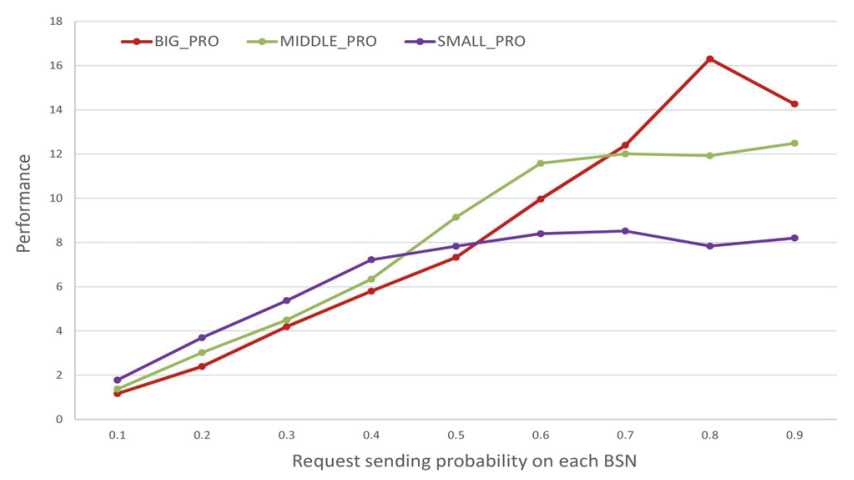

Figure 7: The performances for each protocol with request sending probability on each BSN. that there are least requests in a big node region, which means our probability model is more fit for the heavy workload in the interval network. For the small protocol in Figure 6, the average error, $5.4 \%$, is better than $6.8 \%$ for the big one and $6.0 \%$ for the middle one. Because link utilization and model error are two critical indexes, we define the performance of the model with the ratio of the two indexes: performance $=\frac{\text { linkutilization }}{\text { error }}$.

Figure 7 illustrates that the best performance for the big protocol appears in the probability range of $[0.8,0.9]$. The best performance for middle protocol has a wider probability range of $[0.6,0.9]$, however, it only obtains about $75 \%$ performance of the big one. For the small protocol, the widest best-performance probability range, $[0.4,0.9]$, only provides about half of the performance of the big one. When the request sending probability is less than 0.4, small protocol always takes a better performance than the other two ones.

As the discussion in Section 5, the model uses the matrices of request and transmitting steps to depict the real-time signal path distribution. That means both of the matrices can change the signal distribution on cloud. Hence, we design a simple method to control the final signal distribution through applying a function on the elements in the transmitting matrix $\mathbf{P}$, as shown in Section 5. We find that, if the request matrix is fixed with constants, the final signal distribution is shaped only by the transmitting matrix. For simplification, we choose $1 / N$ for all elements of the request matrix $\mathbf{Q}$, where $N$ is the node quantity in a region. For the transmitting matrix $\mathbf{P}$, we design a set of distribution functions $\left[f_{0}(t), f_{1}(t), \cdots, f_{N-1}(t)\right]$ so that $\sum_{i=0}^{N-1} f_{i}(t)=1$ for any time of $t$. Then, we set $P$ 's elements $p_{i, j}=f_{j}(t)$. Based on the above design, we can prove that the final signal distribution is completely copied from the distribution of the function set.

In order to unveil more detail of the execution of the model, we study another case with more realistic signals. In this case, we monitor the request from one of a $2 \times 2$ ring node regions and control the request sending probability with the method above. We also design a set of distribution functions, $f_{1}(t)=$ $-(1 / 5000) t+1, f_{2}(t)=(7 / 50000) t$, and $f_{3}(t)=(3 / 50000) t$, in a 


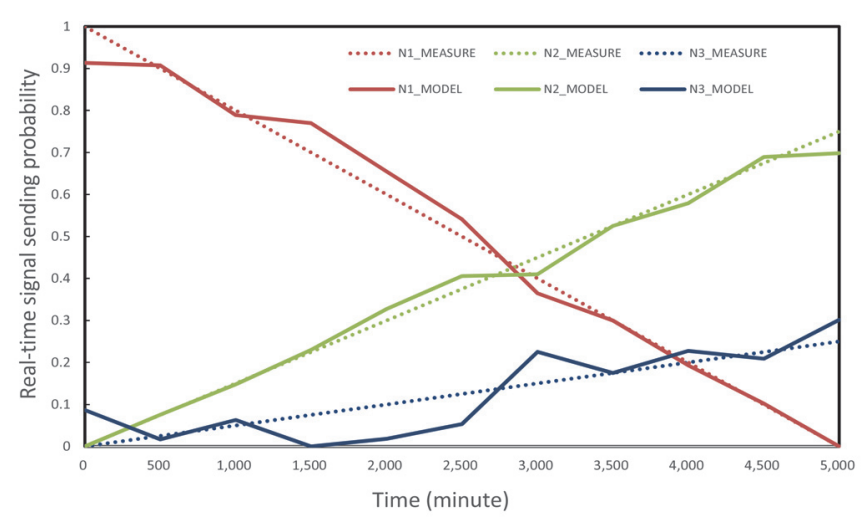

Figure 8: A case: the accuracy of real-time signal sending probability distribution.



Figure 9: The errors of each estimating algorithms.

time domain from 0 to 5000. These three functions satisfy the condition $\sum_{i=1}^{3} f_{i}(t)=1$. The rest node works as cloud manager to deliver the request to the nodes $1-3$ with the probability distribution from the function set. Figure 8 shows the accuracy of our model on reflecting the traffic distribution. The dashed line shows the probability controlled by the distribution function set, and the solid line gives the sampled model values for the three node. In the time domain of [1500, 2500], the node 3 suffers a great error which comes from two reasons, a) we add a random influence to $N_{1}$ node, which leads to a traffic burst at the 1500 th minute $b$ ) the absolute value of the node 3 is quit small which is easy to be affected by other node. An accepted error occurs for node 1 , however this error disturbs the node 3 massively. During the following time, our model is able to recovery from the bursting interruption gradually.

\subsection{Comparing and Optimization of Traffic Estimation}

In this section, we compare the estimation algorithms in Section 6. As the Figure 5 shown, the cloud manager is fed with multiple real-time signals from different remote BSNs. These signals are distributed to several data centers which keep the most recent PHR information of the patients. We use the greedy way which allocates enough bandwidth between the cloud manager and a target data center. To record the current traffic load, cloud manager monitors the flow-rate of the link to the target

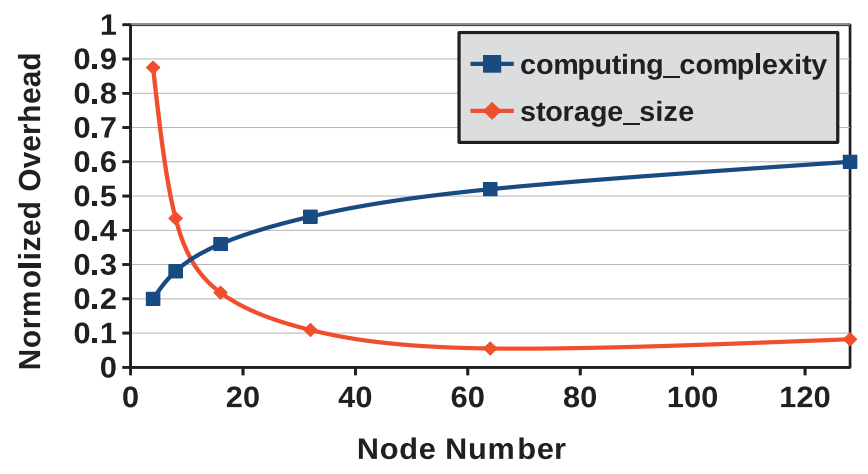

Figure 10: The trade-off between computing and storage with node number scaling up.

data center node periodically so that an 8-step traffic load information is collected to implement the bandwidth prediction. In order to make the simulation approach the real situation, we control those BSNs to send the real-time signal with continuously changing probability.

Figure 9 illustrates the error rate of each bandwidth estimating algorithm in Section 6. The AP algorithm gives a highest error rate of $31.2 \%$. The main reason is that $\mathrm{AP}$ algorithm is more fit to the stable probability. However, our simulation always changes the request rate which make the error unacceptable. The WP and RP algorithm have error rates of $14.6 \%$ and $13.7 \%$, respectively. For the WP algorithm, the recent bandwidth allocation can affect the prediction much more than the old one. For the RP algorithm, the added random item, as shown in Table 1, is also able to decrease the error comparing with the AP algorithm. The most efficient prediction comes from the linear LSP algorithm which only has an error rate of $3.97 \%$. As a continuously changing probability, the linear LSP depicts the data trend for 8 points, which gives an accurate bandwidth estimation.

The AP prediction is a linear method that removes the trend of workload variation and cannot match to the unstable workload from BSN. Thus, its performance is the lowest one among the four predictions. WP and RP features the similar performance which is better than AP by half reduced error rates. The mainly reason is that they both can partially keep the trend of the BSN variation by, 1) for WP, increasing the weight of the close data points, and 2) for RP, stimulating the right predicting values. Finally, we use LSP to achieve the best performance since LSP is able to linearly keep the variation trend of BSN and the " $\geq 2$ " ordered trend is removed.

We also test the scalability of the DCs in Fgure 10 and find that there is a trade-off between the storage and computing overhead. The results shows that, when the node number is increasing from 4 to 128 , the computing workload of single node is increasing. The reason is that the searching procedure in Algorithm 1 is applied to a larger space size which results in an more intense computing overhead. However, the scaling-up leads to lighter storage overhead in single node since there are more nodes used to share the storage workload. 


\section{Conclusions and Future Work}

In this paper, we design a distributed data managing framework for telehealth system, which includes BSN, cloud system, and remote hospital end. By analyzing the features of data processing with medical applications, we provide a decentralized data coherence protocol to solve the performance problems by current design. Our model measures the bandwidth consumption between any node pair in cloud so that the bandwidth can be calculated in each interval. The experimental results show that the bandwidth predicting error is limited in $10 \%$, which provides cloud with a flexible methods (4 types of predicting algorithms) to estimate the bandwidth resources to nodes. Furthermore, a case study shows that our method is able to support finding the most appropriate bandwidth-estimating algorithm for underlining telehealth applications.

In future, we plan to apply our approach to many real-world projects and get the feedback from the collaboration with hospitals. Furthermore, we will work on this topic with more advanced approach, such as hidden Markov model to enhance the performance bandwidth estimating.

\section{Acknowledgments}

This work was supported in part by the State Key Program of National Natural Science Foundation of China under Grant No.61332001, the National Natural Science Foundation of China under Grant No. 61272104 and 61472050, and the Applied Basic Research Program of Sichuan province under Grant No. 2014JY0257. Prof. Qiu is partially supported by NSF 1359557 , NSF 1457506 NSF, and the Open Research Project of the State Key Laboratory of Industrial Control Technology, Zhejiang University, China (No.ICT1441).

[1] T. Shany, S.J. Redmond, M.R. Narayanan, and N.H. Lovell. Sensorsbased wearable systems for monitoring of human movement and falls. IEEE Sensors Journal, 12(3):658-670, 2012.

[2] Pengwei Wang, ZhiJun Ding, Changjun Jiang, and Mengchu Zhou. Design and implementation of a web-service-based public-oriented personalized health care platform. IEEE Transactions on Systems, Man, and Cybernetics: Systems, , 43(4):941-957, July 2013.

[3] Jiayin Li, Meikang Qiu, Zhong Ming, Gang Quan, Xiao Qin, and Zonghua $\mathrm{Gu}$. Online optimization for scheduling preemptable tasks on iaas cloud systems. IEEE Transactions on Parallel and Distributed Systems, 72(5):666-677, May 2012.

[4] M. Ramanathan, Wei-Yun Yau, and Eam Khwang Teoh. Human action recognition with video data: Research and evaluation challenges. IEEE Transactions on Human-Machine Systems, 44(5):650-663, Oct 2014.

[5] Duckki Lee, A.S. Helal, Yunsick Sung, and S. Anton. Situation-based assess tree for user behavior assessment in persuasive telehealth. IEEE Transactions on Human-Machine Systems, 45(5):624-634, Oct 2015.

[6] Xuyun Zhang, Laurence T. Yang, Chang Liu, and Jinjun Chen. A scalable two-phase top-down specialization approach for data anonymization using mapreduce on cloud. IEEE Transactions on Parallel and Distributed Systems, 25(2):363-373, February 2014.

[7] Ming Li, Shucheng Yu, Yao Zheng, Kui Ren, and Wenjing Lou. Scalable and secure sharing of personal health records in cloud computing using attribute-based encryption. IEEE Transactions on Parallel and Distributed Systems, 24(1):131-143, January 2013.

[8] Ali Sunyaev, Dmitry Chornyi, Christian Mauro, and Helmut Krcmar. Evaluation framework for personal health records: Microsoft healthvault vs. google health. In Proceedings of the 2010 43rd Hawaii International Conference on System Sciences, HICSS, pages 1-10, Washington, DC, USA, 2010. IEEE Computer Society.
[9] Carolyn McGregor. Big data in neonatal intensive care. Computer, 46(6):54-59, June 2013.

[10] Li Xiao, Songquing Chen, and Xiaodong Zhang. Adaptive memory allocations in clusters to handle unexpectedly large data-intensive jobs. IEEE Transactions on Parallel and Distributed Systems, 15(7):577-592, July 2004.

[11] Wes Lloyd, Shrideep Pallickara, Olaf David, Jim Lyon, Mazdak Arabi, and Ken Rojas. Performance modeling to support multi-tier application deployment to infrastructure-as-a-service clouds. In Proceedings of the 2012 IEEE/ACM Fifth International Conference on Utility and Cloud Computing, UCC'12, pages 73-80, 2012.

[12] Meikang Qiu and Edwin H. M. Sha. Cost minimization while satisfying hard/soft timing constraints for heterogeneous embedded systems. ACM Transactions on Design Automation of Electronic Systems, 14(2):25:125:30, April 2009

[13] Mamata Dalui and Biplab K. Sikdar. An efficient test design for cmps cache coherence realizing mesi protocol. In Proceedings of the 16th international conference on Progress in VLSI Design and Test, VDAT, pages 89-98, Berlin, Heidelberg, 2012. Springer-Verlag.

[14] V. Goudar and M. Potkonjak. Fault-tolerant and low-power sampling schedules for localized basns. IEEE Journal on Emerging and Selected Topics in Circuits and Systems, 3(1):86-95, 2013.

[15] Peter Leijdekkers, Valerie Gay, and Elaine Lawrence. Smart homecare system for health tele-monitoring. In Proceedings of the First International Conference on the Digital Society, ICDS'07, pages 3-6, 2007.

[16] Steven A. Demurjian, Solomon Berhe, Jaime A. Pavlich-Mariscal, and Alberto De la Rosa Algarin. A security framework for xml schemas and documents for healthcare. In Proceedings of the 2012 IEEE International Conference on Bioinformatics and Biomedicine Workshops (BIBMW), BIBMW'12, pages 782-789, 2012.

[17] Chintan Patel, Karthik Gomadam, Sharib Khan, and Vivek Garg. Invited paper: Trialx: Using semantic technologies to match patients to relevant clinical trials based on their personal health records. Web Semant., 8(4):342-347, November 2010.

[18] Yanzhen Qu and Naixue Xiong. RFH: A resilient, fault-tolerant and highefficient replication algorithm for distributed cloud storage. In Proceedings of the 2012 41st International Conference on Parallel Processing, ICPP, pages 520-529, 2012.

[19] Tommaso Cucinotta, Fabio Checconi, and et. al. Virtualised e-learning on the irmos real-time cloud. Service Oriented Computing and Applications, 6(2):151-166, June 2012.

[20] I. Pavlovic and D. Miklavcic. Web-based electronic data collection system to support electrochemotherapy clinical trial. IEEE Transactions on Information Technology and Biomedicine, 11(2):222-230, March 2007.

[21] Fei Hu, Shruti Lakdawala, Qi Hao, and Meikang Qiu. Low-power, intelligent sensor hardware interface for medical data preprocessing. IEEE Transactions on Information Technology and Biomedicine, 13(4):656663, July 2009.

[22] IEEE draft standard for health informatics - personal health device communication - device specialization - basic electrocardiograph(ECG). IEEE P11073-10406/D07, February 2011, pages 1-69, 2011.

[23] Huang Lin, Jun Shao, Chi Zhang, and Yuguang Fang. Cam: Cloudassisted privacy preserving mobile health monitoring. IEEE Transactions on Information Forensics and Security, 8(6):985-997, June 2013.

[24] J. A. Gutierrez, M. Naeve, E. Callaway, M. Bourgeois, V. Mitter, and B. Heile. IEEE 802.15.4: a developing standard for low-power low-cost wireless personal area networks. IEEE Network: The Magazine of Global Information Exchange, 15(5):12-19, September 2001.

[25] B. P. L. Lo, S. Thiemjarus, R. King, and G. Z. Yang. Body sensor network - a wireless sensor platform for pervasive healthcare monitoring. In Proceedings of the 3rd International Conference on Pervasive Computing (PerCom'05), Kauai, Hawaii, USA, May 2005.

[26] E. Nemati, M.J. Deen, and T. Mondal. A wireless wearable ECG sensor for long-term applications. IEEE Communications Magazine, 50(1):3643, 2012.

[27] Rodrigo N. Calheiros, Rajiv Ranjan, Anton Beloglazov, C\&\#x00e9sar A. F. De Rose, and Rajkumar Buyya. Cloudsim: a toolkit for modeling and simulation of cloud computing environments and evaluation of resource provisioning algorithms. Software: Practice and Experience, 41(1):23-50, January 2011.

[28] Nick Antonopoulos, Nik Bessis, and Stelios Sotiriadis. Exploring inter- 
cloud load balancing by utilizing historical service submission records International Journal of Distributed Systems and Technologies, 3(3):7281, July 2012.

[29] Daniel Warneke and Odej Kao. Exploiting dynamic resource allocation for efficient parallel data processing in the cloud. IEEE Transactions on Parallel and Distributed Systems, 22(6):985-997, June 2011.

[30] C. Jayalath, J. Stephen, and P. Eugster. From the cloud to the atmosphere: Running mapreduce across data centers. IEEE Transactions on Computers, 63(1):74-87, Jan 2014.

[31] Ashok Chandrasekar, Karthik Chandrasekar, Harini Ramasatagopan, Rafica A.R., and Jagadeesh Balasubramaniyan. Classification based metadata management for hdfs. In Proceedings of the 2012 IEEE 14th International Conference on High Performance Computing and Communication, HPCC '12, pages 1021-1026, 2012.

[32] Jihe Wang, Meikang Qiu, and Bing Guo. High reliable real-time bandwidth scheduling for virtual machines with hidden markov predicting in telehealth platform. Future Generation Computer Systems, 49:68-76, 2015 . 